\title{
Auxiliary Plasma Heating and Fueling Models for Use in Particle Simulation Codes
}

\author{
Richard J. Procassini \\ Nuclear Engineering Department \\ University of California \\ Berkeley. CA 94720
}

\author{
Bruce I. Cohen \\ Magnetic Fusion Energy Disision \\ Lawrence Livermore National Laboratory \\ Livermore, $C A 94550$
}

March 14, 1989

\begin{abstract}
Computational mndels of a radiofrequency (RF) heating system and neutratbearu injector are presented. These physics packages. when incorporated into a particle simulation code, allow one to simulate the auxiliary heating and fueling of fusion plasmas. The RF-heating package is based upon a quasilinear diffusion equation which describes the slow esciution of the heated particle distribution. The neutral-team injector package models the charge exchange and impact ionization processes which transfer energy and particles from the beam to the background plasma. Particle simulations of an RF-heated and a neutral-beam-neated simpleinirrcir plasilua aíe presented.
\end{abstract}

\section{DISCLAIMER}

\footnotetext{
This report was prepared as an account of work sponsored by an agency of the Uniled Slates Government. Neither the United States Gnvernment nor any agency thereal, nor any of their employees, makes any warranty, cxpress or implied, or assumes any legal 'iability or responsibility for the accuracy, completeness, or usefulness of any information, apparatus, product, or process disclosed, or represents that its use would not infringe privately owneo rights. Refer. ence herein to any specific commercial product, process, or service by trade name, trademark, manufacturer, or otherwise does not necessarily constitute or imply its endorsement, recom. mendation, or favoring hy the United States Government or any agency thereaf. The views and opinions of authors expressed herein do not necessarily state or reflect these of the United States Government or any agency thereof.
} 


\section{Introduction}

Plasma heating and fueling are two important aspects of controlled fusion research using magnetically confined plasmas. Partial heating of the plasma is possiole by means of collisional (resistive) processes as a current flows through the conducting plasma. The resistivity of the plasma varies as its ten:perature to the negative $3 / 2$ power. Therefore, as the temperature of the plasma is raised, Ohmic heating becomes less effective. For this reason, auxiliary heating methods have been developed. The most effective means of auxiliary heating have been the injection of neutral particle beams and radiofrequency (RF) waves into the plasma. The first method uses $\epsilon$ nergetic neutral atoms to pass through the applied magnetic field into the plasma, where they are subsequently ionized. In the second case, RF waves are injected into the plasma to accelerate and heat the particles.

The most significant difficulty in tueling the plasma is the confining magnetic field, which reduces the leakage of charged particles from the plasma, but also prevents charged fuel particles from entering the plasma. Cne solution to this problem has been the injection of neutral atoms across the coufining magnetic field in to the plasma, where they are ionized by charge exchange and impact ionization processes. Currently, the most widely used forms of plasma fueling are gas puffing, neutral bean: injection and frozen-gas pellet injection. The RF-heating package that was developed for use in the TESS code is described in Sec. 2 of this paper, while the nektral beam charge exchange and ionizasion models are discussed in Sec. 3.

\section{The Radiofrequency (KF) Heating Model}

Particle confinement in mirror plasmas is based upon the conservation of kinetic energy and the adiabaticity of the magnetic moment. such that the particles bounce back and forth in the magnetic potential well. Only a certain fraction of the particles are trapped or confined by this potential well. The trapped region in $r_{\perp}$ vs. $u_{\| l}$ space is defined by the mirror magnetic field atio and the plasma ambipolar electrostatic potential. The main classical ioss mechanism for particles in both simple mirror plasmas and in the end plugs of tandem mirror plasmas is collisional detrapping of the particles from this trapped region into the loss cone. Once in the loss cone, the particles are free to transit out the ends of the device, unhindered by the 
magneti: field.

lons in the center-zell region of a tandern mirror device are electrostatically confined by large positive ambipolar potentials which arise in the end plugs of the device. In the simplest model. this potential increases linearly with the electron temperature in the end plugs

It is possible to improve particle confinement in mirror devices through the injection of ra. diofrequency waves at the fundamental or some harmonic of the electron cyclotron freqeuency. This process is termed electron cyclotron resonance heating (ECRH). The increase in the confinement of center cell ions in a tandem mirror is due to the heating of the plug electrons. which results in an increase in the confining electrostatic potential in the plugs. Of secondary interest is the enhancement of electron confinement in a simple mirror or tandem mirror plug, which is due to the preferential incrase of the perpendicular velocity relative to the parallel velocity. Thus leads to hot electrons which are placed farther into the trapped region of velocity space. thereby requiring a longer time to collistonally migrate to the loss boundary in velocity space. This assumes that the hot electrons remain microstable.

\section{$2.1 \quad$ Methodology}

The quasilinear RF-heating model of Bernstein and Baxter [1], which treats the heating of mir-or-trapped particles, forms the basis of the RF-heating package used in TESS. It is valid for mildy rela:ivistic electrons and nonrelativistic ions in the presence of a cyclotron resonant applied electromagnetic field. The model, based upon the so-called "perturbed-distribution" method (see [4]), assumes that the cyclotron frequency is much higher than tile bounce frequency ii, the magnetic patential well, and then averages over the particle trajectorics as they bounce in the well. One of the inain results of Reference [1] is the derivation of a quasilinear diffusion equation in the form of a Fokker-Planck equation. This diffusion equation describes the slow evolution of the heated particle distribution function $f_{o}$ in $\varepsilon$ (total energy), $\mu$ (magnetic moment) space.

The Bernstein-Baxter formulaticin of the quasilineat diffusion equation was used by Roglien in the Monte Carlo RF-heated mirror code MCPAT $[2,3]$ and generalized formally by Smith and Cohen [A]. That implemantation of the model was cranslated by Bruce Cohen for use in TESS The model is based upon the "perturbed-trajectory" method in action $(J)$ - angle $(0)$ space. 
where $J$ and $O$ are constants of the particle motion. Since we are interested in long-tume-scale (relative to the "correlation" time $t_{c}$, cyclotron orbit time $t_{L}$, mirror bounce time $t_{b}$, etc.). averaging is performed ove: the angle sarmables, resulting in the Fokker-Planck equation for the slcwly varying heated distribution function $f_{0}$

$$
\frac{\partial f_{0}}{\partial t}=-\frac{\partial}{\partial J}\left(\Gamma_{J} f_{0}\right)+\frac{\partial^{2}}{\partial J^{2}}\left(D_{J J} f_{0}\right)
$$

where the coefficient of dynamic friction and the diffusion coefficient are given by

$$
\begin{aligned}
\Gamma_{J} & =(\Delta J / \Delta t\rangle \\
D_{J J} & =\left\langle(\Delta J)^{2} /(2 \Delta t)\right\rangle .
\end{aligned}
$$

Applying the friction-diffusion relation

$$
\Gamma_{J}=\frac{d D_{J J}}{d J}
$$

reduces the Fokker-Planck equation (1) to the form of a standard diffusion equation

$$
\frac{\partial f_{0}}{\partial t}=\frac{\partial}{\partial J}\left(D_{J J} \frac{d f_{0}}{d J}\right)
$$

For $t$ is particular implementation of the model, the action variable $J$ was chosen to be the total particle energy $\varepsilon$, which is a constant of the motion for unheated mirror-trapped particles.

The RF-heating model was incorporated into the particle code in the following way. The region over which the RF power is being injected is defined by a center location $Z_{\text {oRF }}$ and a width $\Delta Z_{R F}$. The intensity of the injected $R F$ power varies within the region as

$$
E_{R F}(z)=\frac{E_{R F}\left(Z_{o_{R F}}\right)}{2}\left[1+\cos \left(\frac{2 \pi\left(z-Z_{o_{R F}}\right)}{\Delta Z_{R F}}\right)\right]
$$

where $E_{R F}\left(Z_{\text {ORF }}\right)$ is the maximum amplitude of the $\mathrm{RF}$ wave within the region. Particles which lie within this region are tested to determine if they meet the resonance condition $\mathbf{R}=0$, where

$$
\mathbf{R}=\omega_{R F}-\frac{\ell \Omega_{s}}{\gamma}-\frac{k_{\|} p_{\|}}{\gamma m_{s}}
$$

with $\omega_{R F}$ the frequency of the injected wave, $\ell$ the cyclotron harmonic number, $\Omega_{3}=q, B / \mathrm{m}, \mathrm{c}$ the cyclotron Frequency for species $s_{,} \uparrow=1 / \sqrt{1-v^{2} / c^{2}}$ the relativistic factor, $k_{\|}$the wave vector paralle] to the magnetic field, and $p_{\|}=\gamma m$ s $v_{\|}$the particle mornentum parallel to the magnetic field. Finally, $m$, and $q$, are the species rest mass and charge respectively, and $c$ is the 
speed of light. If the value of $\mathbf{R}$ changes sign in one time step. the particle has gone through resonance, and is therefore given a "kick" in energy

The change in energy that the resonant particle receives is given by

$$
\begin{aligned}
\Delta \varepsilon & =\Gamma_{\varepsilon} \Delta t+\left(6 D_{\varepsilon \varepsilon} \Delta t\right)^{1 / 2} R_{N} \\
& =\left(\frac{d D_{c \varepsilon}}{d \varepsilon}\right) \Delta t+\left(6 D_{\varepsilon \varepsilon} \Delta t\right)^{1 / 2} R_{S}
\end{aligned}
$$

where $s=\gamma m_{s} c^{2}+q_{s} \Phi$ is the total particle energy for an electrostatic potential $\Phi . \Delta t$ is the time step and $R_{N^{\prime}}$ is a uniformly distributed random number in the range $[-1.1]$. The coefficient of dynamic friction $\Gamma_{\varepsilon}$ from (2) and the energy diffusion coefficient $D_{t e}$ from (3) are specified functions of the particle rest mass, charge, parallel velocity, magnetic monnent, gyroradius, cyclotron frequency and the parallel and perpendicular wave vectors (see [1]). The derivative of the energy diffusion coefficient with respect to total energy may also be written as

$$
\frac{d D_{\varepsilon \varepsilon}}{d \varepsilon}=\frac{\partial D_{\varepsilon \varepsilon}}{\partial \varepsilon}+\alpha \frac{\partial D_{\varepsilon \varepsilon}}{\partial \mu}
$$

where $\alpha=\left(\mathrm{i}-k_{\|} v_{\|} / \omega_{R F}\right) / B$ and the magnetic moment $\mu=\gamma^{2} \mathrm{mg}_{1}^{2} / 2 B$.

The first term on the right side of (9) represents the change in total energy due to dynamic friction or drag, while the second term represents the change due to diffusion in $\varepsilon, \mu$ space. These are the same two processes that are described by the right side of the Fokker-Planck equation (1). Note that the energy increment $\Delta \varepsilon$ may be either positive or negative, such that the particle gains energy from the wave. or vice versa.

Once the total energy jncrement is known, we calculate the change in the particle parallel momentum and magnetic moment via

$$
\Delta p_{\|}=\gamma m_{,} c^{2} \frac{k_{\|} v_{\|}}{\omega_{R F}} \frac{\Delta \varepsilon}{p_{\|} c^{2}}
$$

and

$$
\Delta \mu=\frac{\gamma}{B}\left(1-\frac{k_{\| v_{\|}}}{\omega_{R F}}\right) \Delta \varepsilon=\frac{\gamma}{B} \alpha \Delta \varepsilon
$$

\subsection{Simulation Results}

In addition to the data required to run the basic electrostatic particle code, TESS also requires the following data for the RF heating model: the center and width of the RF heated region 
$\left(Z_{o_{R F}}\right.$ and $\left.\Delta Z_{R F}\right)$, the wave frequency $\left(\mathcal{H}_{R F}\right)$, the cyclotion harmonic mumber of thr wave $(\mathcal{E})$. the peak RF wave amplitude $\left(E_{R F}\left(Z_{O_{R F}}\right)\right)$ and the parallei anf perpendicular wave vectors $\left(k_{\|}\right.$ and $\left.k_{1}\right)$.

Results of the TESS code are compared to those from MCPAT below. For these runs. the magnetic field profiles were specified in both codes, and the self-consistent calculation of the ambipolar electrostatic potential was disabled in TESS, in an effort to more closely compare with MCPAT, which does not have this capability. The runs are therefore simulations of a simple mirror geometry, with ECRH applied at the bottom of the magnetic well. The RF waves are injected essentially perpendicular to the magnetic field at the bottom of the magnteic well, with a small component along the magnetic field. The input data for the runs were $Z_{\text {oRF }}=250 \mathrm{~cm}$, \lrcorner $Z_{R F}=50 \mathrm{~cm}, \omega_{R F}=8.8 \times 10^{10} \mathrm{rad} / \mathrm{sec}, E_{R F}\left(Z_{o_{R F}}\right)=3 \mathrm{~V} / \mathrm{cm}, \ell=1$ or fundamental heating, and $k_{1}=1.0 \mathrm{~cm}^{-1}$ and $k_{\|}=0.1 \mathrm{~cm}^{-1}$.

The election velocity-space plots for the RF heated region from TESS and MCPAT are shown in Figure 1. Note the increase in particle density in the regions of high $v_{1}$ and low $v_{\|}$, which is the indication of preferential heating in the $\perp$-direction, relative to the $\|$-direction. The initial thermal velocity in each direction is $v_{t h}=4.19 \times 10^{8} \mathrm{~cm} / \mathrm{sec}$, such that the most energetic particles have been heated to a maximum velocity $v_{\perp_{\max }} \simeq 6 v_{t h}$.

The kinetic energy profiles along the axis of the simple mirror are shown in Figure 2. Note that the TESS result is an instantaneous snapshot, while the MUPAT result is time-averaged. The $\mathrm{MCPAT}$ profile from $-150 \mathrm{~cm}$ to $150 \mathrm{~cm}$ corresponds to the region from 100 to $400 \mathrm{~cm}$ (the peaks of the magnetic field) on the TESS profile. Note that in each case, the parallel kinetic energy is, on average, half that of the perpendicular kinetic energy. This is becatse the perpendicular direction comprises two degrees-of-freedom, as compared with one for the parallel direction. The heating is manifested almost entirely in the increrse of the perpendicular kinetic energy in the $25 \mathrm{~cm}$ to either side of the injection center point. TESS gives a maximum perpendicular kinetic energy of $215 \mathrm{eV}$, while MCPAT yiejds a $200 \mathrm{eV}$ maximum value. The large values of the perpendicular kinetic energy near the peaks of the magnetic fieid in the TESS results are due to particles which are near their tarning points, with large values of $v_{\perp}$ and nearly zero values of $v_{\|}$. This effect is not seen in the MCPAT results due to the time-averaging of the profile. 


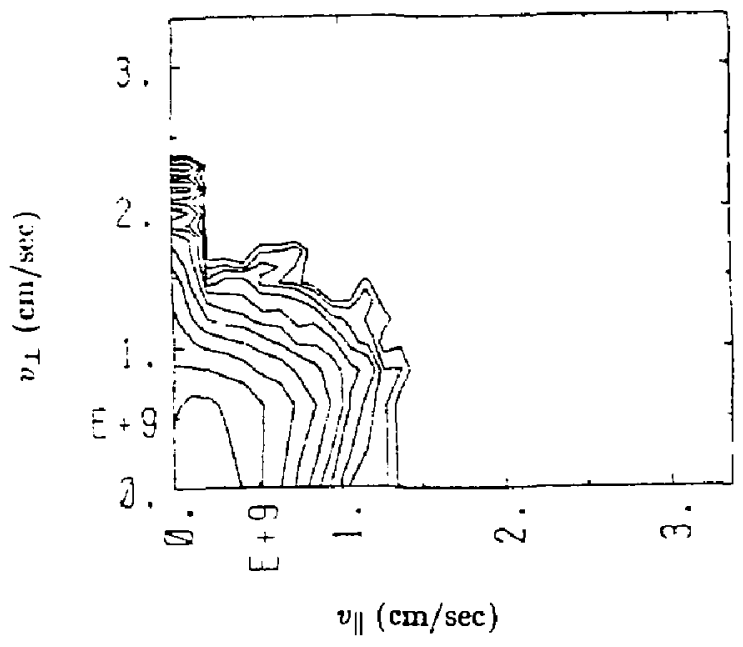

a) TESS velocity space density plot.

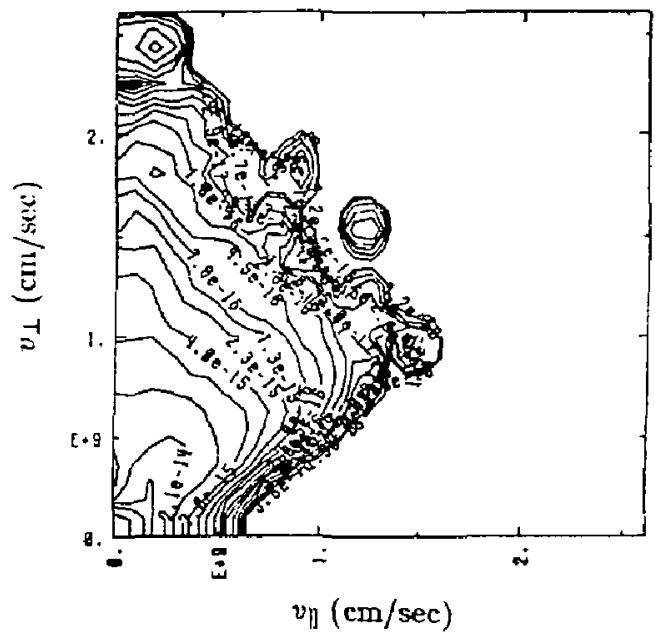

b) MCPAT velocity space density plot.

Figure 1: Electron $v_{\downarrow}, v_{\|}$velocity space density plots from (a) TESS and (b) MCPAT for the region near the bottom of the magnetic well, showing the preferential increase in perpendicular velocity due to heating by appljed electromagnetic waves. 


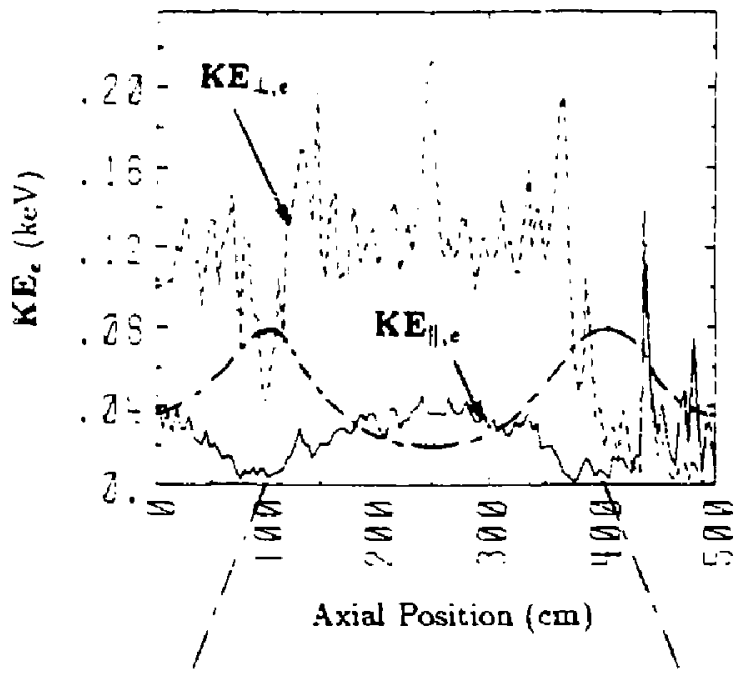

a) Instantaneous kinetic energy profile from TESS.

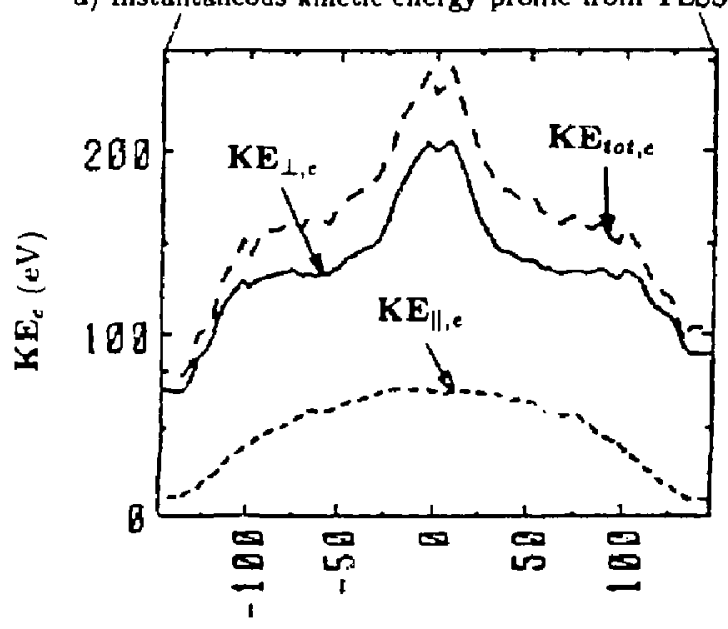

Axial Position (cm)

b) Time-averaged kinetic energy profile from MCPAT.

Figure 2: Electron kinetic energy profiles from (a) TESS and (b) MCPAT showing the increase in perpendicular kinetic energy in the RF-heated region. The RF-heated region is $25 \mathrm{~cm}$ on either side of the magnetic well minimum. 


\section{The Neutral Beam Injection Model}

The most common method of auxiliary plasma heating presently in use is the injection of energetic neutral particles into the plasma. Heating of the plasma results as these neutrals undergo charge exchange with plasma ions, or are ionized via collisions with the plasma particles. Note that the former mechanism results only in a change in the total particle "nergy, while the latter also results in an increase in both the ron and electron densities. For this reason, neutral beam injection (NBI) may also be used as a means of fueling the plasma.

Neutral beam injection has been successfully employed for several years to both heat and fuel plasmas in experimental configurations ranging from tokamaks to stellarators to mirror machines. In addition, current-drive schemes which rely on NBI have been proposed ior use in the ignited. steady-state tokamaks of the future $[5,6]$. Two additional uses of NBI are found in tandem mirror devices with thermal barriers. The first is the use of "sloshing" ions in the formation of the thermal barriers. Since the sloshing ions (which are created by injection of neutral beams at some angle $\theta<90^{\circ}$ to the axis of the machine) are mirror trapped, the averige density at the turning points of these particles in the magnetic well is larger than that at hise center of the welk, which in turn leads to a potential minimum near the center of the vell. This poiential dip is necessary to the formation of a thermal barrier which isolates the hot electrons in the flug region from their colder counterparts in the central cell. The second use of NBI in tandeli mirrors is to maintain the thermal barrier, by charge exchiuge "pumping" of the cold, passing ions which become collisionally trapped in the thermal barrier. Lastly, NBI may be used to improve particle confinement in mirror devices via injection of particles in a direction perpendicular to the axis of the machine, such that the injected particles are located sufficiently far from the loss boundary in velocity space.

\subsection{Methodology}

The neutral-beam-injection package in the TESS code is used to reproduce the effects of charge exchange and impact ionization of the beam as it passes through the background plasma. The code allows for the use of two separate neutral-beam injectors. For each injector, one must specify the center location $Z_{O_{N B I}}$ and width of the beam $\Delta Z_{N B I}$. The beam intensity varies 
over this region as

$$
I_{N B I}(z)=\frac{I_{N B I}\left(Z_{O_{N B I}}\right)}{2}\left[1+\cos \left(\frac{2 \pi\left(=-Z_{O_{N B I}}\right)}{\Delta Z_{N B I}}\right)\right]
$$

where $I_{N B I}\left(Z_{O N B I}\right)$ is the peak beam intensity. Additionally, the code requires the usn to input the mean energy of the beam $\epsilon_{N B I}$, the beam thermal energy spread $k T_{N B I}$, the angle of injection $\theta_{N B}$ relative to the parallel direction, the neutral beam current $\mathcal{I}$ and the rates of charge exchange $R_{C X}$ and impact ionization $R_{l}$. An examination of the time scales in fusionrelevant plasmas indicates that the charge exchange and ioniza',ion times are of the order of the collisional relaxation times, which are much longer than other physical tinues (cyclotrori orbit time $t_{L}$, mirror bounce time $t_{b}$, etc. ). For this resion, the rates $R_{C X}$ and $R_{l}$ ar a multiplied by a collision rate "acceleration" factor. "The method of determining the velocity components of the injected particles is discussed in the following two subsection-

\subsubsection{The Charge Exchange Model}

A charge exchange event occurs when a neutral atom transfers one or more electrons to an adjacent ion. This proces; results in a charge in the kinetic energy of the charged and neutral species, with the charged and neutral particle Jensities remaining constant. If the initial neutral atoms have kinetic energies which are larger than those of the initial ions, the overall effect is heating of the ions at the expense of the neutral atoms. This $i$, the basis of n utral-bean heating of a plasma.

The charge exchange model that is incorporated into the TESS code is similar to one used previously by Cohen, et. al. [7] in a study of the stabilization of ion-cyclotron microinstabilities in electrostatic mirror devices. The region over which charge exchange is to take place is divided up into grid cells. The number of ions in a given cell $j$ which are to undergo charge exchange per time step is determined by

$$
N_{C X}=\min \left[N_{j} R_{C X}, N_{C X_{\text {mas }}}\right]\left(I_{N B I,}+I_{N B I_{y+1}}\right) / 2
$$

where $N_{j}$ is the number of ions in grid cell $j_{1} I_{N} g I$, is the relative beam iatensity for grid cell $j$ in the range $[0,1]$ and $N_{C .:}$ mo: is the maximum number of charge exchange processes allowed per time step, which is set by the beam current

$$
N_{C X_{m a x}}=\frac{I \Delta t}{q}
$$


for a neutral beam current $\mathcal{I}$ of ions with charge $q$. (Th: model assumes that the injected atoms are accelerated, prior to neutralization. at a charge state that is the same as that of the prasma ions.) The minimum function in (14) ensures that the charge exchange rate will not be larger than that associated with ine beam current $I$. $N_{C x}$, ions will undergo a charge exchange evem In grid cxell $j$ if $\hat{N}_{C x}>1$. If $N_{c x},<1$, a single charge exchange event will take place only if $V_{c x} \geq R_{N}$, wher $R_{N}$ is a random number in the range $(0,1)$. The $N_{C X}$, plasma ions in grid cell $j$ that are to undergo charge exchange are chosen randomly from the list.$N$,

The velocity components $v_{\perp}$ and $\ddot{u}_{i j}$ are modified as a result of the charge exchange event; however, the position of the initial and final ion is the same. The velocity components of the $n_{t} *$ ion are dependent upon the mean energy $c_{V B I}$ and thermal spread $k T_{N B I}$ of the neutral beam. of well as the angle of injection $\theta_{\mathrm{NB1}}$. The therral spread is assumed to be isotropic and Maxwellian in Cartesian velocity space, such that the spread about the mean velocity of the bearn i: fiven by

$$
\Delta v=\left[\left(2 \Delta \epsilon_{i / B I} / m_{n}\right) \ln \left(\frac{1+\delta}{1+\delta-R_{N}}\right)\right]^{1 / 2}
$$

: there $\delta=10^{-8}$ and $R_{N}$ is a random number in the range $(0,1)$ and $m_{n}=m_{i}+m_{e}$ is the mass of the neutral atom. The argument of the logarithm ranges in value from 1 to $10^{-8}$, representing the limits on the value of the normalized distribution function $f(\Delta v)$. The velocity spread in each direction is given by $\Delta v_{x}=\Delta v \cos \left(\theta_{1}\right) \sin \left(\theta_{2}\right), \Delta v_{y}=\therefore v \sin \left(\theta_{1}\right) \cos \left(\theta_{2}\right)$ and $\Delta v_{z}=\Delta v \cos \left(\theta_{2}\right)$, where $\theta_{1}$ is randomly chosen from the interval $[0,2 \pi]$ and $\theta_{2}$ is rantdomly chosen from $[0, \pi]$. The mean velocity comprnents of the beam are defined as $\left\langle v_{x}\right\rangle=$ $\langle v\rangle \sin \left(\theta_{N B I}\right) \cos \left(\theta_{3}\right),\left\langle v_{y}\right\rangle=\langle v\rangle \sin \left(\theta_{N B I}\right) \sin \left(\theta_{3}\right)$ and $\left\langle v_{s}\right\rangle=\langle v\rangle \cos \left(\theta_{N B I}\right)$ where the average beam velacity is

$$
\langle v\rangle=\sqrt{2 \epsilon_{N B I} / m_{n}}
$$

and $\theta_{3}$ is randomly chosen from $[0,2 \pi]$. The model also assum ${ }^{-\infty}$ that particles are injected in the positive and negative $\|$ or $\Sigma$ direction in equal ismbers. Therefore, the resultant injected particle velocity components are $v_{x}=\left\langle v_{x}\right\rangle+\Delta v_{x}, v_{y}=\left\langle v_{y}\right\rangle+\Delta v_{y}$ and $v_{\|}=v_{z}= \pm\left(\left\langle v_{z}\right\rangle+\Delta v_{z}\right)$. Finally, note that $v_{1}=\sqrt{v_{x}^{2}+v_{y}^{2}}$. These velocity components are then used to calculate the relativistic factor $\gamma$, the parallel moment $\lrcorner$ In $p_{z}$ and magnetic moment $\mu$ for the $i: a$.

\subsubsection{The Impact Ionization Model}

Neutral atoms thay also be ionized via collisions with the charged farticles of the plasma. 
If the charged particles strike the atoms with sufficient force, one or more electrons will be stripped from the neutral atom. For atoms which are isotopically identical to the plasma ions. this process results in an increase in the plasma electron and in density. When an energetic neutral beam is injected into the plasma, the overall effect is an increase in both the temperature and density of the plasma.

The impact ionization model is based upon the charge exchange algorithm which is described in the previous paragraphs. The number of electron-ion pairs that are to be injected into a given cell $j$ per time step is

$$
N_{l,}=\min \left[N_{j} R_{l}, N_{I_{\text {mas }}}\right]\left(I_{N B I},+i_{N B I_{j+1}}\right) / 2
$$

with $N_{I_{\max }}$ the maximurn number of particle pairs injected into grid cell $j$ by a neutral beam of current $\mathcal{I}$

$$
N_{I_{\mathrm{ma}}}=\frac{I \Delta t R_{I}}{q R C X}
$$

For the case $N_{I_{\text {max }}}<1$, the method employed in the charge exchange model is also used here.

The electron-ion pairs are injected at the same position, which is chosen randomly in the grid cell. The velocity components of the neutral atom are calculated in the same manner as those of the new ion in the charge exchange model. A major assumption of this model is that the velocity components of the injected electron and ion are the same as those of the injected reutral atom. This means that there will be no electrostatic source sheath created as a result of the injection of the electron-ion pairs, since $v_{\|_{e}}=v_{\|_{1}}$ (i. e. no separation of the injected charge). The injected particle velocity components are used to calculate the relativistic factor $\gamma$; the parallel momentum $p_{z}$ and magnetic moment $\mu$, which are then written into the permanent particle arrays. If the number of particles injected per time step exceeds the free space at the end of the permanent particle arrays, the arrays are dynamically expanded by the input variable $N_{\text {ezpand }}$. In order to reduce the amount of time spent expanding the arrays, $N_{\text {erpand }}$ is chosen such that there are of order 100 time steps between sucessive array expansions.

\subsection{Simulation Results}

The characteristics of the charge exchange and impact ionization models are demonstrated via the simulation of a neutral-beam-heated simple mirror plasma. The neutral beam is injected 
perpendicular to the magnetic field at the hottom of the magnetic well. The high energy ( $. v B I=I \mathrm{keV})$, warm $\left(k T_{N B I}=250 \mathrm{eV}\right)$ neutral beam impinges upon a low cemperature plasma $\left(k T_{e}=k T_{i}=50 \mathrm{eV}\right)$ of mass ratio $m_{i} / m_{e}=100$. The other input data values for the run are $Z_{N B I}=180 \mathrm{~cm}, \Delta Z_{N B I}=100 \mathrm{~cm}, G_{N B I}=90^{\circ}, I=0.225$ Statcoulombs $/ \mathrm{sec}, R_{C X}=0.020$ and $R_{l}=0.007$. The ratio $R_{C x} / R_{l}$ is chosen to be the ratio of the charge exchange to impact ionization cross-sections, which are a function of the beam energ: plasma temperature and mass of the injected atom [8].

Since the ionization model assumes that the injected electrons and ions bave the same velocity components, the injected electron kinetic energy is approximately $m_{e} / m_{i}$ times that of the ion. For large mass ratios, the ion kinetic energy is effectively the beam energy. In addition. the charge exchange process leads only to an increase in the ion kinetic energy. Therefore, one expects to see substantial heating of the ions and only minimal heating of the electrons.

The kinetic energy profiles for the ions and electrons are shown in Figure 3. The dashed and solid lines represent the perpendicular and parallel kinetic energy profiles respectively. (The dash-dotted line is the profile of the magnetic field intensity.) Note that for each of the species, the kinetic energy components are minimized near the peaks in the magnetic field intensity, resulting from the dereased particle densities in these regions. Figure 3a shows that the ions are the principal recipients of the injected energy, with a peak perpendicular kinetic energy of $\varepsilon_{\perp, i_{\text {mas }}} \simeq 1 \mathrm{keV}$ and a peak parallel kinetic energy of $\epsilon_{\|, i_{\text {max }}} \simeq 100 \mathrm{eV}$. The fact that the neutral beam was injected perpendicular to the magnetic field lines accounts for the large ratio of the perpendicular to parallel kinetic energies. The peak perpendicular and parallel electron kinetic energies (Figure $3 \mathrm{~b}$ ) are $\epsilon_{1, e_{\mathrm{max}}} \simeq 50 \mathrm{eV}$ and $\epsilon_{\|, c_{\text {mas }}} \simeq 25 \mathrm{eV}$ respectively. (Note that the kinetic energy of a species is one-half the species temperature.) The effect of neutral-beam injection on the electrons has been a doubling of the perpendicular kinetic energy. with almcat no increase in the parallel kinetic energy. These results are consistent with the expectations of the previous paragraph. The ion perpendicular kinetic energy profile (Figure 3a) shows the effect of the spatial variation of the beam intensity on the heating of the particles. The peak intensity of the beam lies at $z=180 \mathrm{~cm}$.

The species perpendicular-velocity phase space scatter plots are shown in Figure 4. Again we note that the ions are heated to a much larger degree than are the electrons. Figure $4 a$ indjcates that the injected ions have peak perpendicular velocities of $v_{\perp, i \text { max }} \simeq 11 v_{t h \perp, i}$. (The 


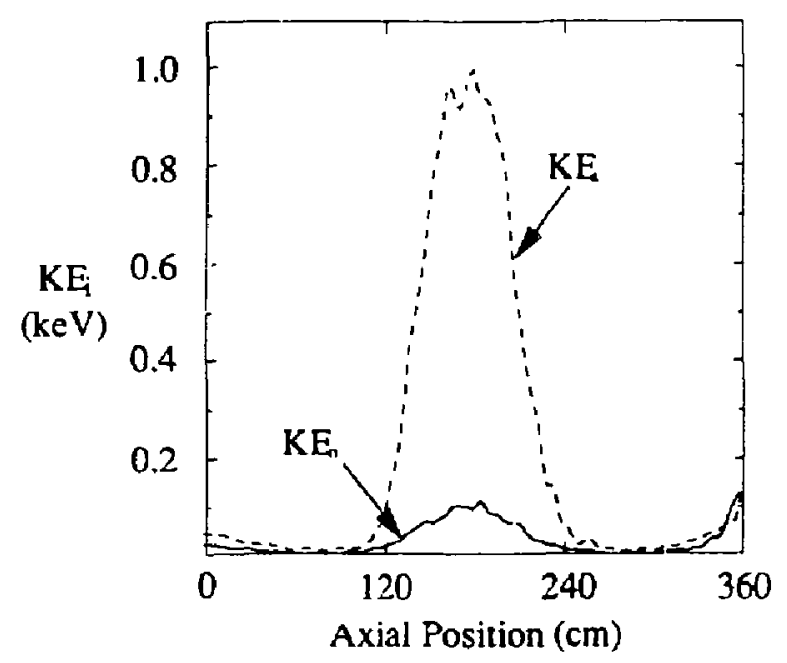

a) Ion kinetic energy profiles.

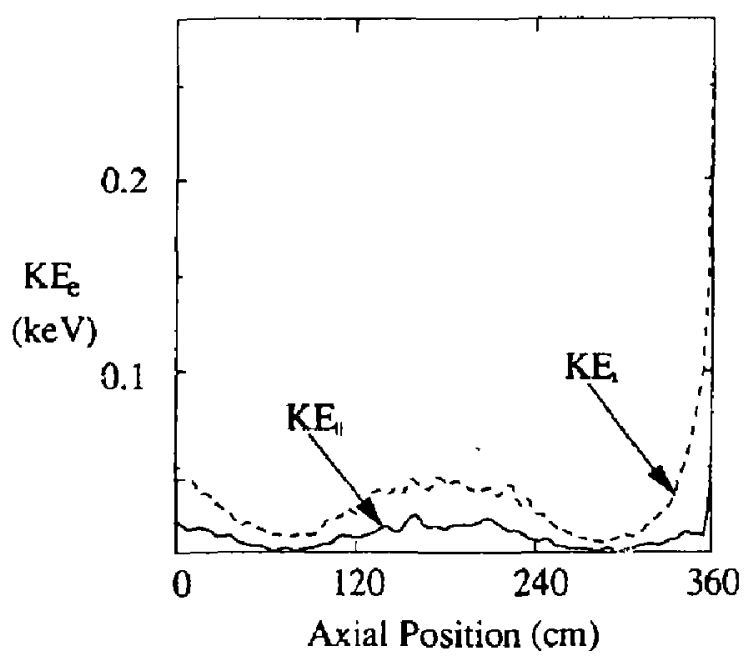

b) Electron kinetic energy profiles.

Figure 3: Profiles of the (a) ion and (b) electron perpendicular and parallel kinetic energy profiles for the case of a neutral beam heated simple mirror plasma. The beam is injected perpendicular to the magnetic field lines at the bottom of the magnetic well. 
solid horizontal lines represent the species thermal velocity). The injected electrons have peak perpendicular velocities that are oniy $\simeq 10 \%$ larger than those in the background Maxwellian distribution.

Finally, the species velocity-space plots for the spatial tegion between the two magnetic feld peaks are presenced in Figure 5. Figure 5a clearly shows the injected ions (with large values of $v_{\perp}$ ) and the background Maxwellian ions. Note the large population of injected ions that lie above the background population, with $v_{\perp} \gtrless 1.25 \times 10^{8} \mathrm{~cm} / \mathrm{sec}$. The wide range of $v_{\|}$-space that they occupy is due to the fact that the injected ions have finite values of $v_{\|}$, hence they bounce in the magnetic well. The electron velocity-space plot (Figure 5 b) shows only a small deviation from a Maxwellian as a result of the neutral beam injection.

\section{Acknowledgments}

We are grateful to T. D. Rognlien for sharing with us the details of his Monte Carlo model of RF heating and for providing general assistance at various stages of this project. This work was performed under the auspices of the U. S. Department of Energy by the Lawrence Livermore Vational Laboratory under contract No. W-7405-Eng-48.

\section{References}

[1] 1. B. Bernstein and D. C. Baxter, Phys. Fluids, 24, 108 (1981).

[2] T. D. Rognlien, Phys. Fluids, 26, 1545 (1983).

[3] T. D. Rognlien, Nucl. Fusion, 23, 163 (1983).

[4] G. R. Smith and B. I. Cohen, Phys. Fltids, 26, 238 (1983),

[5] D. E. Post and the ITER Physics Team, Bull. Am. Phys. Soc., 33 (9), 1972 (1988).

[6] R. S. Devoto, M. E. Fenstermacher, G. R. Smith, P. T. Bonoli and M. Porkolab, Bull. Am. Phys. Soc., 33 (9), 1901 (1988).

[7] B. I. Cohen, G. R. Smith, N. Maron and W. M. Nevins, Phys. Fluids, 26, 1851 (1983).

18] A. C. Riviere, Nud. Fusion, 11, 363 (1971). 


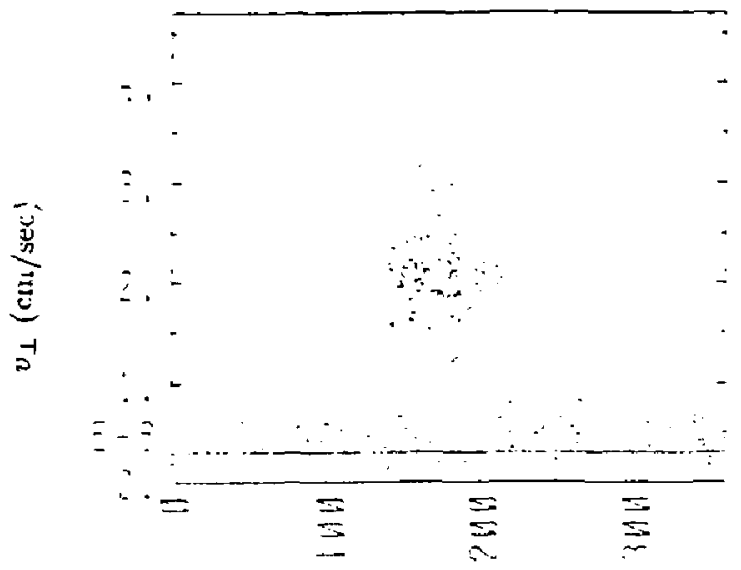

Axial Position (cm)

a) Ion perpendicular-velocity phase space.

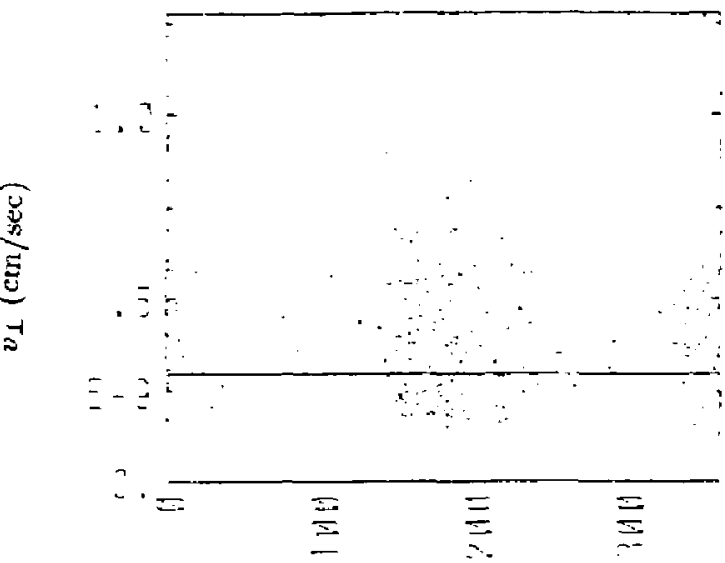

Axial Position (cm)

b) Electron perpendicular-velocity phase space.

Figure 4: Perpendicular-velocity phase space scatter plots of the $(a)$ ions and (b) electrons for the case of a neutral beam heated simple mirror plasma. The beam is injected perpendicular to the magnetic field lines at the bottom of the magnetic well. 


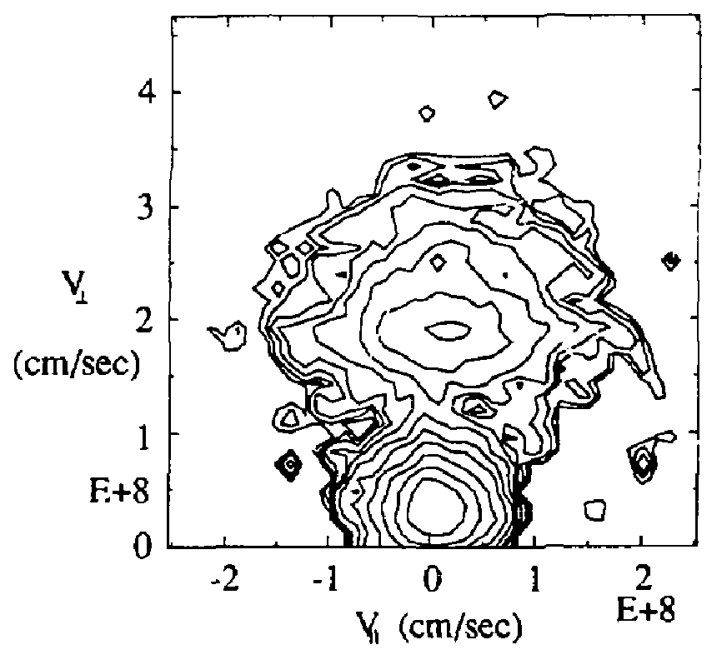

a) Ion $v_{\| l}, v_{\perp}$ velocity space.

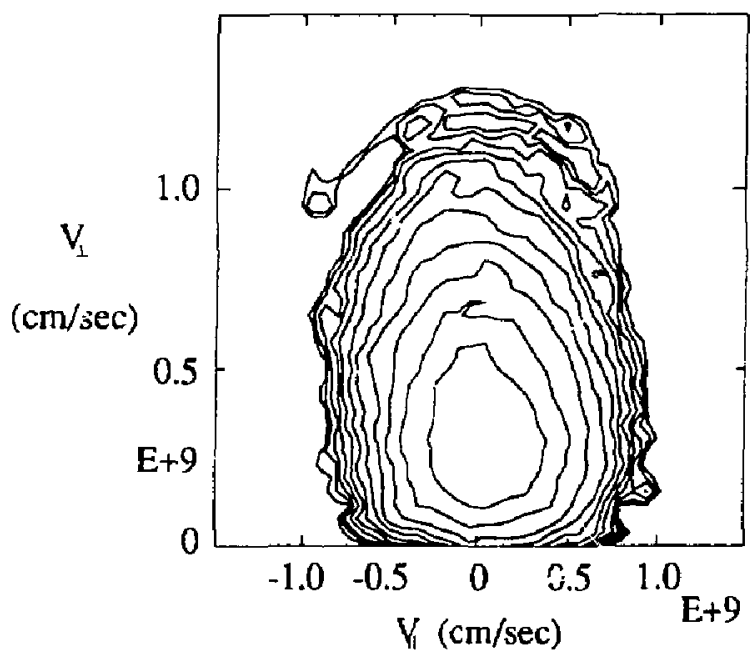

b) Electron $\imath_{\| !}, v_{\perp}$ velocity space.

Figure 5: Velocity space plots of the (a) ions and (b) electrons for the case of a neutral beam heated simple mirror plasma. The beam is injected perpendicular to the magnetic field lines at the bottom of the magnetic well. 